\title{
Agricultural genetics still on ice
}

\section{The exciting prospect that biotechnology will transform the technology of agriculture must, sadly, be moderated by the certainty that progress will be unreasonably delayed.}

THE accelerating progress of plant biotechnology has been paralleled in the United States by rising disquiet in the research community about disproportionate public concern over the environmental impact of genetically manipulated organisms. The consistent success of Jeremy Rifkin in obstructing field trials of even the most trivially modified organism must have contributed to the anxiety apparent at the Du Pont-UCLA Symposium on Molecular Strategies for Crop Protection two weeks ago, a meeting otherwise notable for its ebullience in the light of recent triumphs (a more detailed account of which will appear in a forthcoming issue).

The meeting, held during the Easter week at Steamboat Springs, Colorado, saw the announcement of two important new developments in the genetic manipulation of crop plants. Hitherto, developments have been largely confined to the successful transfer of marker genes of one sort or another, or of genes conferring tolerance to herbicides. What makes the recent advances potentially important is that they involve the transfer of genes that confer protection from natural pests and pathogens. Biologists at Washington University, St Louis, for example, in collaboration with Monsanto, have used the viral gene encoding the coat protein of tobacco mosaic virus to produce limited protection against the subsequent inoculation of tobacco plants with the complete virus. At the same time, both Agrigenetics and Plant Genetic Systems have demonstrated expression, again in tobacco plants, of the gene for the Bacillus thuringiensis toxin, which is lethal to insect pests but harmless to almost everything else.

It is far too early to claim for either of these interesting experiments a place in the history of agricultural practice: although each has come successfully through preliminary tests for efficacy against viruses and insects respectively, greenhouse tests do not reliably predict what will happen in the field; and therein lies the problem. It has taken Agricetus, for example, three years to get permission from the US Department of Agriculture (USDA) and the National Institutes of Health (NIH) simply to test the behaviour in the field of tobacco plants containing nothing more exciting than the vector sequences from the $\mathrm{Ti}$ plasmid that is routinely used in plant gene transfer, along with marker genes of no functional significance and an enzyme marker that makes it possible to keep track of the expression of the transferred genes in the plant. The trials may now take place this summer.

In the meantime, R. Kaufman of Monsanto estimates that it has cost $\$ 2.5$ million in risk assessment to develop a soil bacterium, Pseudomonas fluorescens, expressing the insecticidal $B$. thuringiensis toxin and intended as a means of biological control of insect pests that attack the roots of plants. Subject to the approval of the Environmental Protection Agency (EPA), the modified organism will make a tentative debut this year, contained within a system of fences and buffer zones that seem somewhat excessive for an organism that is incapable of surviving for more than six months in the soil and can be eliminated by the application of household bleach; especially since the new gene it contains specifies a protein that is already in use as a topical insecticide.

The fear is that such costly delays at each stage in the development of a potential new agricultural product may quite quickly deter even the larger companies from investing further in plant biotechnology. The smaller ones could not possibly survive them.

The arguments in favour of some relaxation of the rules, or at least streamlining of the procedures, range from the frankly sanctimonious (the potential benefit to farmers and mankind in general) to the disarmingly candid (it is in the commercial interests of companies to produce strictly obsolescent bugs), and with an estimated one-third of the world's crops going to feed the world's pests and pathogens it is difficult to question the validity of at least the first of these arguments - assuming, of course, as everyone does, that the genetic manipulation of plants will eventually extend beyond tobacco and its kin.

It would be foolish to argue that no precautions should be taken to limit the spread of genetically modified plants and bacteria, or monitor their possible effects. The problem is that in a sense there is no real problem. By and large, neither the organisms nor the introduced genes are harmful and there is therefore no reason to expect any harm from the combination. What Kaufman and his collaborators have done by way of risk assessment is to establish that the modified Pseudomonas is no more toxic than the $B$. thuringiensis that naturally produces the toxin and that the survival of the engineered bacterium is (as was almost bound to be the case) compromised rather than enhanced by the interference with its genome. What Rifkin and his like seem to be demanding is an assessment of some unknown and certainly unspecifiable risk somehow inherent in the fact of artificially recombining DNA - a risk there is simply no rational way of assessing.

The absurdity of this position is most clearly seen in the notorious case of the ice-minus bacterium whose release by the biotechnology company Advanced Genetic Sciences has been successfully prevented so far by Rifkin and whose future, despite the permit recently granted by EPA, is still in doubt (see Nature 319, 254 and $320 ; 1986)$.

The point about this particular experiment is that the genetically modified bacterium has a natural counterpart: although the majority of the natural Pseudomonas syringae organisms produce a protein that nucleates ice crystals (and can hence damage fruit crops), some lack the gene specifying the ice-nucleating protein.

The same gene is deleted in the genetically manipulated bacteria, and thus the only reason for all the fuss is that in the case of the AGS organism the genetic recombination was deliberately engineered in the laboratory.

History suggests that, with time, the fuss will die down. Anaesthetics for childbirth were a crime against the natural order when they were first used by a few medical pioneers. Indeed in Europe, where for the most part public reactions are more muted, field tests involving the (scrupulously monitored) release of manipulated organisms are scheduled for this year. Britain, for example, will experiment with a genetically modified virus whose release into the pine forests of Scotland is expected to help curb the ravages of the moth Panolis flammae (see Nature 320, 2; 1986).

It would be ridiculous to pretend that the protests registered at Steamboat Springs were disinterested; but at the same time it would be a pity if unreasonable restrictions on field testing were to defeat the impetus provided by the interest of industry to fundamental research on important crops such as rice, and important pathogens such as the fungi.

In the end it may be Europe's initiative that saves the day: Americans hate to be left behind.
Miranda Robertson 